\title{
Aberrant Long Noncoding RNAs Expression Profiles Affect Cisplatin Resistance in Lung Adenocarcinoma
}

\author{
Lijuan Hu, ${ }^{1}$ Jian Chen, ${ }^{1}$ Fan Zhang, ${ }^{1}$ Junjun Wang, ${ }^{1}$ Jingye Pan, \\ Jie Chen, ${ }^{2}$ and Yumin Wang ${ }^{1}$ \\ ${ }^{1}$ Department of Laboratory Medicine, The First Affiliated Hospital of Wenzhou Medical University, Wenzhou 325000, China \\ ${ }^{2}$ Department of Intensive Care Unit, The First Affiliated Hospital of Wenzhou Medical University, Wenzhou 325000, China
}

Correspondence should be addressed to Jie Chen; chenjie991300@163.com and Yumin Wang; wym0577@163.com

Received 31 May 2017; Revised 24 August 2017; Accepted 13 September 2017; Published 27 November 2017

Academic Editor: Paul Harrison

Copyright (C) 2017 Lijuan Hu et al. This is an open access article distributed under the Creative Commons Attribution License, which permits unrestricted use, distribution, and reproduction in any medium, provided the original work is properly cited.

Background. Long noncoding RNAs (lncRNAs) have been shown to be involved in the mechanism of cisplatin resistance in lung adenocarcinoma (LAD). However, the roles of lncRNAs in cisplatin resistance in LAD are not well understood. Methods. We used a high-throughput microarray to compare the lncRNA and mRNA expression profiles in cisplatin resistance cell A549/DDP and cisplatin sensitive cell A549. Several candidate cisplatin resistance-associated lncRNAs were verified by real-time quantitative reverse transcription polymerase chain reaction (PCR) analysis. Results. We found that 1,543 lncRNAs and 1,713 mRNAs were differentially expressed in A549/DDP cell and A549 cell, hinting that many lncRNAs were irregular from cisplatin resistance in LAD. We also obtain the fact that 12 lncRNAs were aberrantly expressed in A549/DDP cell compared with A549 cell by quantitative PCR. Among these, UCA1 was the aberrantly expressed lncRNA and can significantly reduce the IC50 of cisplatin in A549/DDP cell after knockdown, while it can increase the IC50 of cisplatin after UCA1 was overexpressed in NCI-H1299. Conclusions. We obtained patterns of irregular lncRNAs and they may play a key role in cisplatin resistance of LAD.

\section{Introduction}

In recent years, a growing proportion of lung adenocarcinoma (LAD) has been diagnosed as non-small cell lung tumor (NSCLC) that is attributable to causes such as environmental pollution. The combination of cisplatin-based chemistry plays an important role in comprehensive treatment program [1]. With the widespread use of cisplatin, tumor cells will inevitably lead to its resistance and the chemotherapy effect was significantly reduced [2]. Studies show that $70-80 \%$ of patients can temporarily be alleviated in the initial stage of chemotherapy, but long-term use of cisplatin leads to $60 \%$ or more of recurrence rate, and drug resistance rate of recurrent lung tumor was significantly increased and chemotherapy response rate was less than 30\% [3]. Nowadays, chemotherapy response rate of patients with advanced LAD was only $30-40 \%$ and five-year survival rate less than $15 \%$ [4]. According to the survey of American Tumor Society, it was shown that more than $90 \%$ of tumor deaths in patients were related to varying degrees of drug resistance. The formation of tumor cells once resistant to cisplatin resulted in multidrug resistance to many first-line chemotherapy drugs, for example, Adriamycin, vinblastine, fluorouracil, and mitomycin, so the harm is particularly serious [5]. Cisplatin resistance is the leading cause of LAD chemotherapy failure, affecting the cure rate and long-term survival rate, seriously affecting the prognosis and quality of life, but also aggravating the social and medical burden. Therefore, it is very important to find the biomarkers and molecular targets related to cisplatin resistance in LAD and then to reverse its resistance to improve the prognosis and to avoid and overcome multidrug resistance.

Recent studies have shown that cisplatin is a nonspecific cell cycle cytotoxic drug; it mainly plays a role of inhibition of tumor cell DNA synthesis [6], inducing apoptosis [7]. The mechanism of cisplatin resistance is very complex and it mainly involved some mechanisms [6, 8-10], including changes in intracellular drug transport (such as ATP binding 
cassette protein); reducing drug activity interfering with drug action mechanisms such as glutathione (such as GST-pi) can increase cell detoxification function and affect DNA damage repair (breast tumor-associated gene 1 , excision repair crosscomplementing gene). Genetic changes of the main signal pathways (PDK/Akt, MAPK/Erk, and Wnt) lead to block apoptosis of drug effects. Unfortunately, despite previous advances in genomics and proteomics, the mechanism of cisplatin resistance has not been elucidated.

Studies have shown that lncRNAs known to be aberrantly expressed in normal cells and tumor cells play a role in the regulation of gene expression; so irregular expression of lncRNAs can result in abnormalities of gene expression and tumorigenesis [11-19]. The abnormal expression of lncRNAs is a symbol of many tumors and has been shown to further the development, invasion, and metastasis of tumors by a variety of mechanisms [20, 21]. LncRNAs can regulate expression from the epigenetic, transcriptional, and posttranscriptional levels [20-22].

It was shown that lncRNAs are related to the mechanisms of resistance to cisplatin in tumors, including lung tumor [23-26], providing an important opportunity to elucidate the mechanisms of cisplatin resistance in tumor cells and to find ways to reverse cisplatin resistance. At present, the research of 1ncRNA of cisplatin resistance in LAD is still in its infancy. Some lncRNA molecules, including HOTAIR [27], AK126698 [28], MEG3 [23], H19 [29], and ROR [30], have been screened and identified. It is shown that HOTAIR-mediated LAD cisplatin-resistant mechanism may be through the impact of p21 gene expression to enhance cell apoptosis and G0/G1 phase cell cycle arrest [27], AK126698 regulate non-small cell lung tumor cisplatin resistance partially by Wnt signaling pathway [28], MEG3 expression by inducing mitochondrial apoptosis pathway p53 protein, and Bcl-xl activation of A549/DDP cells to reduce cisplatin resistance [23]. However, lncRNAs of LAD cisplatin resistance need to be further excavated and their mechanisms need to be clarified.

We used a high-throughput microarray to compare the lncRNA and mRNA expression profiles in cisplatin resistance cell A549/DDP and cisplatin-sensitive cell A549. Several candidate cisplatin resistance-associated lncRNAs were verified by real-time quantitative reverse transcription polymerase chain reaction (PCR) analysis. Our results suggest that lncRNA expression patterns may provide new molecular biomarkers for the prediction of cisplatin resistance in LAD.

\section{Materials and Methods}

2.1. Cell Lines. A549, NCI-H1299 cells and cisplatin-resistant cell line A549/DDP were cultured in RPMI1640 medium containing $10 \%$ fetal bovine serum. The cells were transferred into cell culture flasks by pipetting. The cells were incubated at $37^{\circ} \mathrm{C}$ in a 5\% CO2 incubator, while A549/DDP cell was added to $1 \mu \mathrm{g} / \mathrm{ml}$ of cisplatin maintain the drug resistance. The medium was changed every 2-3 days. The cells were observed under the good condition and the cell will be digested as the cell density was 70\%-90\%.
2.2. RNA Extraction. The test group included three parallel cultured A549/DDP cell and three parallel cultured A549 cell as the control group. Total RNAs of cells were extracted using Trizol reagent (Invitrogen, Carlsbad, CA, USA), based on the manufacturer's protocol. The integrity of the RNA was analyzed by electrophoresis on a denaturing agarose gel. The accurate measurement of RNA concentration $\left(\mathrm{OD}_{260}\right)$, protein contamination $\left(\mathrm{OD}_{260} / \mathrm{OD}_{280}\right.$ ratio), and organic compound contamination $\left(\mathrm{OD}_{260} / \mathrm{OD}_{230}\right.$ ratio) was analyzed with a NanoDrop ND-1000 spectrophotometer

2.3. Microarray and Computational Analysis. An Agilent Array analysis platform (Agilent Technologies, Santa Clara, CA, USA) was used for microarray analysis,. Slightly, an mRNA-ONLY Eukaryotic mRNA Isolation Kit (Epicentre Biotechnologies, USA) purified mRNA from total RNA after removal of rRNA. Then, each sample was amplified and transcribed into fluorescent cRNA along the whole length of the transcripts without 39 bias with a random priming method. The labeled cRNAs were hybridized onto a Human LncRNA Array v3.0 (8660 K; Arraystar including 30,586 lncRNAs and 26,109 coding transcripts). A specific exon or splice junction probe accurately identified each transcript. For hybridization quality control, the positive probes for housekeeping genes and negative probes were also printed onto the array. The arrays were scanned with an Agilent G2505C scanner after washing the slides, and Agilent Feature Extraction software (version 11.0.1.1) was used to analyze the acquired array images. Quartile normalization and subsequent data analysis were performed with the GeneSpring GX v12.0 software package (Agilent Technologies). Complying with the manufacturer's standard instructions with minor modifications, sample preparation and microarray hybridization were performed [31]. The microarray study was performed by KangChen Bio-tech Corporation, Shanghai, China.

2.4. Functional Group Analysis. GO analysis was derived from Gene Ontology (http://www.geneontology.org), which can provide three structured networks of defined terms describing gene product attributes. The $P$ value hints the significance of GO Term enrichment in the unmorally expressed mRNA list ( $P \leq 0.05$ was considered obviously significant). We also performed pathway analysis for the unmorally expressed mRNAs based on the latest KEGG (Kyoto Encyclopedia of Genes and Genomes) website. This analysis results allowed us to determine the biological pathways whether a significant enrichment of unmorally expressed mRNAs existed ( $P \leq 0.05$ was considered obviously significant).

2.5. Construction of the mRNA-lncRNA Gene Coexpression Network. The coexpression network is constructed and used with calculating a pairwise relation matrix between all probe sets across microarray samples. The result of Pearson relation matrix was transformed into an adjacency matrix [31].

2.6. Quantitative PCR. Total RNA was then reversetranscribed with an RT Reagent Kit (Thermo Scientific, CA, USA), according to the manufacturer's protocols. Total RNA 
TABLE 1: LncRNAs gene primers in the study for qPCR.

\begin{tabular}{lccc}
\hline LncRNA gene & \multicolumn{1}{c}{ Sense primer $\left(5^{\prime}-3^{\prime}\right)$} & Antisense primer $\left(5^{\prime}-3^{\prime}\right)$ & PCR product length $(\mathrm{bp})$ \\
\hline GLYCTK & CGTGCTGATCTCAGGTGGTGA & CTTCACAGAACGTGGCAGGG & 164 \\
HSD17B7P2 & GTCAGCAACCTGCAGTCATTC & GAGGCTCCAGTTCCCGAATC & 283 \\
AP001469.9 & TCACACAACCACATCTCGTG & TTGGTCTAAGACTGTTGCCAAG & 299 \\
NABP1 & GGAGGGTGGGAAGCTTTGAC & CTCCGATCTCATCCCACACG & 260 \\
RP11-909N17.3 & AGACCCCTGCTATTCCCAGT & AAGGGATGCAGGCAGTTCTC & 102 \\
UCA1 & ACGCTAACTGGCACCTTGTT & CTCCGGACTGCTTCAAGTGT & 124 \\
POLD4 & GCACCGTCTCTGGCATCTC & GTTGAGCCTCTGACACCTCC & 180 \\
XLOC_009833 & AGCCCCTTTATCACTGTGGC & GACATTCAGGAGACGACGGG & 147 \\
CTD-2555O16.2 & GAGAGAAGGTCCCTTGGTGC & CAGTGCTGCGTTTAGTCATGT & 79 \\
RP11-299H22.5 & AGTCGCCTTTTCCCTTAGCC & GCAGCTCTCATCTGGTGCTT & 108 \\
BC033241 & TCTACACAACGCCAGCACAT & TTGACACGTGCTTGGTGAGA & 107 \\
AC078883.3 & GTGGCAACATCCCTACACCA & ACAGGTTCGTGTTCCCAGTC & 245 \\
GAPDH & TGACTTCAACAGCGACACCCA & CACCCTGTTGCTGTAGCCAAA & 121 \\
\hline
\end{tabular}

(2 ug) was reverse-transcribed to cDNA. PCR was performed in a total reaction volume of $20 \mu \mathrm{l}$, including $10 \mu \mathrm{l}$ of SYBR Premix $(2 \mathrm{x}), 2 \mu \mathrm{l}$ of cDNA template, $1 \mu \mathrm{l}$ of PCR forward primer $(10 \mathrm{mM}), 1 \mu \mathrm{l}$ of PCR reverse primer $(10 \mathrm{mM})$, and $6 \mu \mathrm{l}$ of double-distilled water. The qPCR reaction included an initial denaturation step of $10 \mathrm{~min}$ at $95^{\circ} \mathrm{C} ; 40$ cycles of $5 \mathrm{~s}$ at $95^{\circ} \mathrm{C}, 30 \mathrm{~s}$ at $60^{\circ} \mathrm{C}$, and a final extension step of $5 \mathrm{~min}$ at $72^{\circ} \mathrm{C}$. The qPCR detected LncRNA with SYBR Premix Ex Taq and an ABI 7200 instrument (ABI Corporation, CA, USA). The candidate lncRNAs were analyzed by qPCR and the sequence information of these gene primers is shown in Table 1 . We verified the expression of these lncRNAs by qPCR using GAPDH as a housekeep gene and by calculating $2^{-\Delta \Delta C T}$ values [32].

\subsection{Lentivirus-Mediated siRNA, Overexpression Vector Con-} struction, and Transfection. We constructed siRNA GV248 vector targeting UCA1 and overexpression GV303 vector targeting UCA1 (Gene Chem, Shanghai, China). SiRNA sequences were as follows: siRNA1: CCACCTGTAGAGAAGACAAA, siRNA2: GAAGAGTAGAAG ACAGGT, siRNA3: GCCTGGACAAGAACAGT. Transfections were performed by seeding $2 \times 10^{5}$ cells in 6 -well plate. After $24 \mathrm{~h}$, the medium was replaced, and the cells were incubated with the transfection complex based on the manufacturer's protocol; the multiplicity of infection (MOI) values was as follows: A549 MOI $=20$ and NCI-H1299 MOI $=5$. The cells were infected with lentivirus for $72 \mathrm{~h}$, and the siRNA or overexpression efficiency was assessed by qPCR. Puromycin test isolated these cell lines successfully transfected with the lentivirus-mediated vector. The study included NCI-H1299 UCA1 overexpression cell lines (UCA1 OE group), NCI-H1299 was infected with lentivirus negative control LVCON077 vector (NC group), NCI-H1299 (control group), and A549 UCA1 siRNA cell line (UCA1 siRNA group), and A549 was infected with lentivirus negative control LVCON145 vector (NC group) and A549 (control group).

2.8. CCK-8 Assays. IC50 (half maximal inhibitory concentration) was detected by Cell Counting Kit-8 (CCK-8, Corning
Corporation, UAS) abiding by the manufacturer's protocols. Briefly, 3000 cells were remixed and seeded into a 96-well plate with $10 \%$ FBS. The next day, the cells were incubated with CCK- 8 for $1 \mathrm{~h}$ and the absorbance of $450 \mathrm{~nm}$ was analyzed.

2.9. Statistical Methods. Statistical analysis was performed for the comparison of two groups in the microarray which was performed with Student's $t$-test and the fold change. The false discovery rate (FDR) was calculated for correcting the $P$ value. The threshold value used to designate abnormally expressed lncRNAs and mRNAs according to a fold change of $\geq 2.0$ or $\leq 0.5(P<0.05)$. Differences with $P<0.05$ were considered statistically significant.

\section{Results}

3.1. Overview of $\ln R$ RA Profiles. The result showed that there were 1,543 differentially expressed lncRNAs between A549/DDP and A549 cell. Among these, compared to A549 group, the 984 lncRNAs upregulated more than twofold in the A549/DDP group, while 559 lncRNAs downregulated (Supplemental Table 1 in Supplementary Material available online at https://doi.org/10.1155/2017/7498151 and Figure 1). These lncRNAs might play an important role in the cisplatin resistance of $\mathrm{LAD}$.

3.2. LncRNA Classification and Subgroup Analysis. There were 43 differentially intergenic lncRNAs (LincRNAs) (including 31 upregulated and 12 downregulated) expressed (fold change $\geq 2.0, P<0.05$ ) between A549/DDP cell and A549 cell. We also found some nearby coding genes that may be regulated by these LincRNAs (Supplemental Table 2). LncRNAs with enhancer-like functions (lncRNA-a) were identified with GENCODE annotation. There were 33 lncRNA-a (including 17 upregulated and 16 downregulated) differentially expressed between A549/DDP and A549 cell. We also found some nearby coding genes that may be regulated by these IncRNA-a (Supplemental Table 3 ). Otherwise, we also found 52 antisense lncRNAs (including 


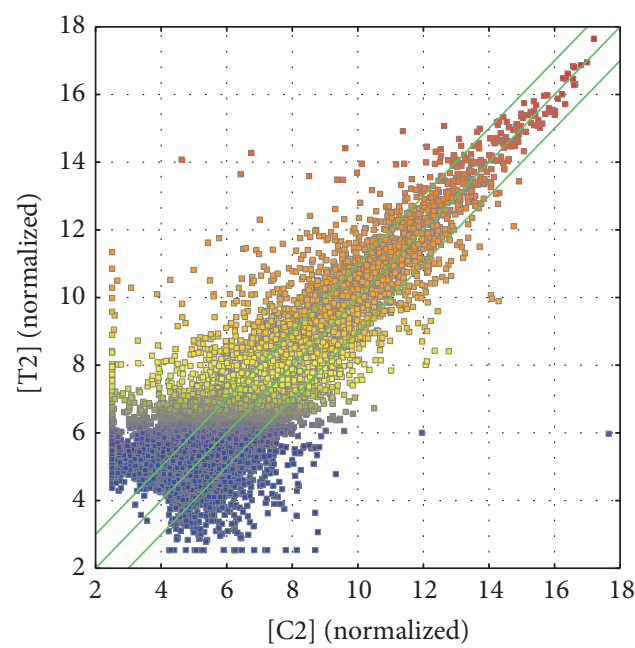

(a)

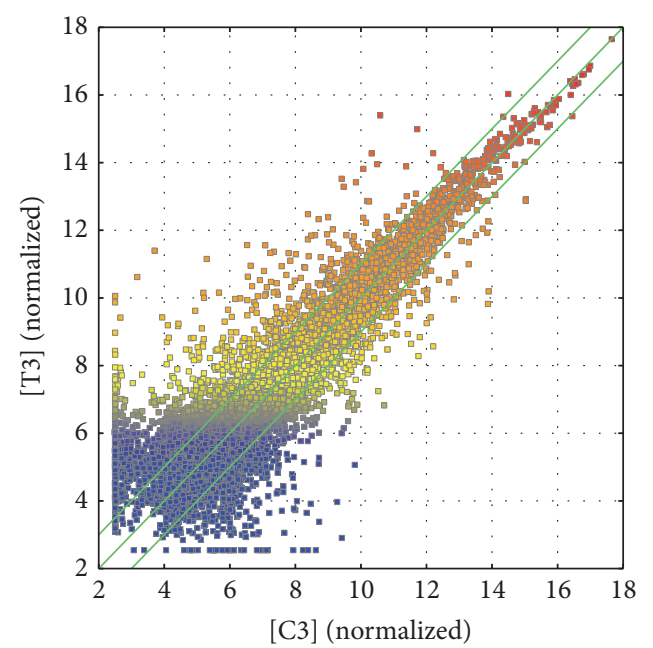

(b)

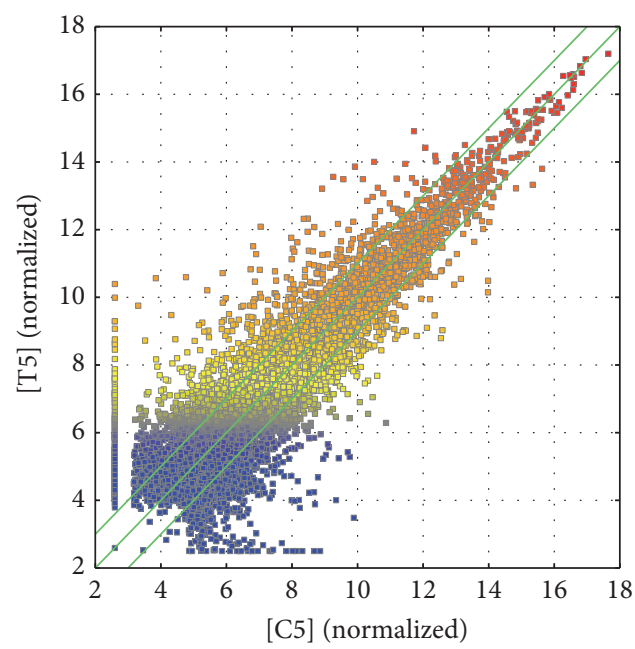

(c)

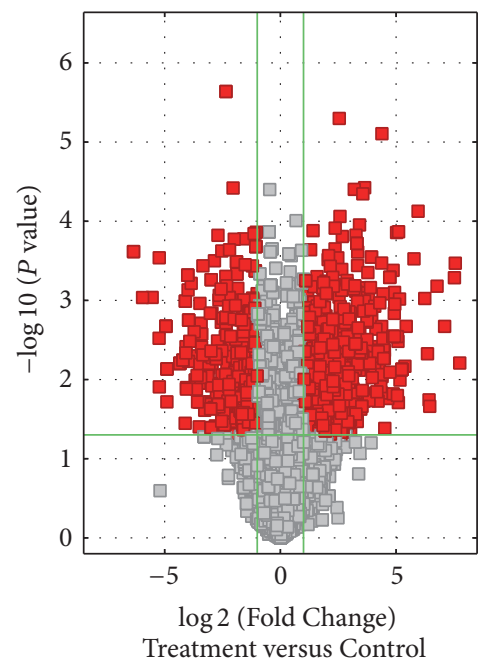

(d)

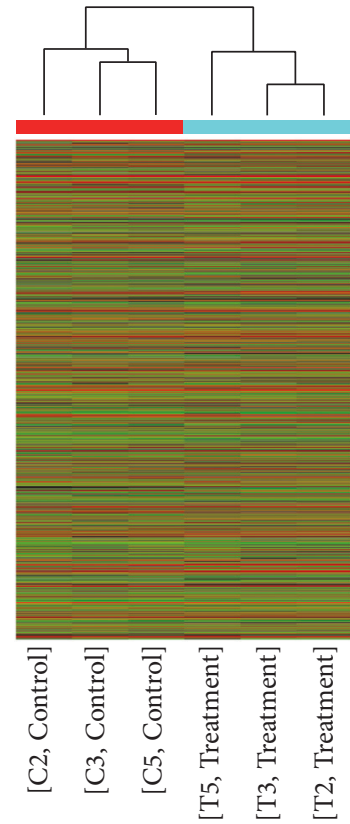

(e)

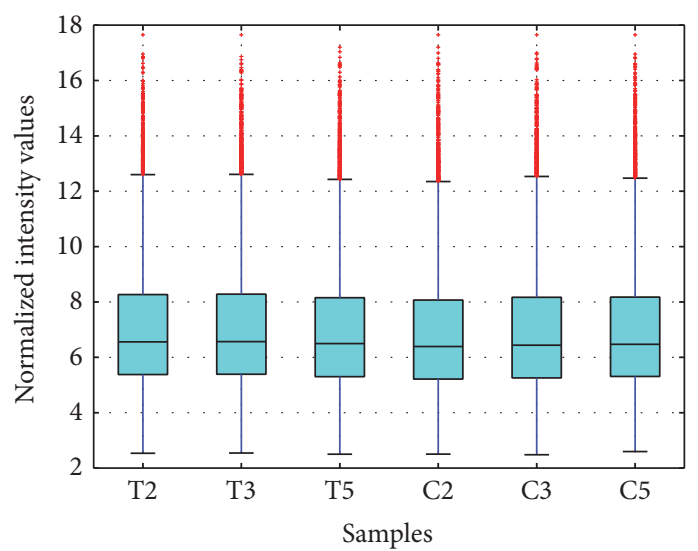

(f)

FIGURE 1: Box plots, scatter plots, and heat map showing the variation in lncRNA expression between the A549/DDP and A549 arrays. The values of the $X$ and $Y$ axes in the scatter plot are averaged normalized values in each group ( $\log 2$-scaled). The lncRNAs above the top green line and below the bottom green line are those with a $>3$-fold change in expression between tissues. (a) Scatter plots of T2 group versus C2 group. (b) Scatter plots of T3 group versus C3 group. (c) Scatter plots of T5 group versus C5 group. (d) Volcanic map, (e) heat map and hierarchical clustering of lncRNA, and (f) box plots showing the distribution of the lncRNA. 
TABLE 2: Some upregulated or downregulated mRNAs in A549/DDP.

\begin{tabular}{lccc}
\hline Probe name & Fold change & Regulation & Gene symbol \\
\hline ASHGA5P001180 & 39.0517312 & up & USF2 \\
ASHGA5P016060 & 3.9089125 & up & EFTUD2 \\
ASHGA5P005374 & 3.2721382 & up & TTC39C \\
ASHGA5P034316 & 3.2788507 & up & ZNF836 \\
ASHGA5P006930 & 2.1690486 & down & TUBD1 \\
ASHGA5P050222 & 4.1895085 & down & PAFAH1B3 \\
ASHGA5P006058 & 9.1726844 & down & TACC3 \\
ASHGA5P010515 & 2.6021545 & down & QKI \\
ASHGA5P037999 & 2.193611 & down & FAM159A \\
ASHGA5P009559 & 2.2639906 & down & DIAPH2 \\
ASHGA5P001190 & 3.9636124 & up & CPA4 \\
ASHGA5P002598 & 2.1978303 & up & SNX24 \\
\hline
\end{tabular}

21 upregulated and 31 downregulated) (Supplemental Table 4).

3.3. Overview of $m R N A$ Profiles. In total, $1,712 \mathrm{mRNAs}$ were found to be differentially expressed between the A549/DDP and A549 cell, including 795 mRNAs upregulated and 917 mRNAs downregulated (Table 2 and Figure 2). These mRNAs might play a role in the cisplatin resistance of LAD.

3.4. GO Analysis. The genes corresponding to downregulated mRNAs included 979 genes involved in biological processes, 120 genes involved in cellular components, and 137 genes involved in molecular functions (Figures 3(a)-3(c)). The genes corresponding of upregulated mRNAs included 558 genes involved in biological processes, 93 genes involved in cellular components, and 77 genes involved in molecular functions (Figures 3(d)-3(f)).

3.5. Pathway Analysis. The 30 upregulated pathways were found, including chemical carcinogenesis, drug metabolism, and p53 signaling pathway (Figure $3(\mathrm{~g})$ and Table 3). 37 downregulated pathways were identified, like DNA replication, cell cycle, Fanconi anemia pathway, and so on. (Figure 3(h) and Table 3). These pathways might play a role in the cisplatin resistance of LAD.

3.6. LncRNA-mRNA Coexpression Network. We build the lncRNA-mRNA coexpression network. See Figure 4(a). The results imply that UCA1 (uc002nbr.3), ENST00000443252, ENST00000510562, ENST00000565689, ENST00000558690, ENST00000397340, ENST00000440955, ENST00000507916 are closely related to many mRNAs and they together prompted resistance of cisplatin in LAD.

3.7. Real-Time Quantitative PCR Validation. Based on the features (such as fold difference, gene locus, and nearby encoding gene, and so on.) of the differentially expressed lncRNAs, we initially identified a number of interesting candidate lncRNAs for further analysis (including HSD17B7P2, GLYCTK, NABP1, AP001469.9, RP11-909N17.3,
UCA1, POLD4, XLOC_009833, CTD-2555O16.2, RP11299H22.5, BC033241, and AC078883.3). We found that the microarray results for several of the lncRNAs were consistent with the results of RT-PCR (Figure 4(b)). Of these, UCA1 exhibited significantly changed expression in 20 samples from A549/DDP and A549 cell. The expression of UCA1 in cisplatin-resistant A549/DDP cells was significantly higher than that in cisplatin-sensitive A549 cells $(t=71.14$, $P=0.0002$, Figure $4(c))$. These results suggest that UCA1 and candidate lncRNAs may play an important role in cisplatin resistance in LAD.

3.8. UCA1 Significantly Reduces the IC50 of Cisplatin in A549/DDP Cell after Knockdown. We used CCK-8 method to detect the sensitivity of A549/DDP cells to cisplatin. The results showed that the IC50 of A549 was $2.09 \mu \mathrm{g} / \mathrm{ml} \pm$ $0.08 \mu \mathrm{g} / \mathrm{ml}$, IC50 of A549/DDP was $10.7 \mu \mathrm{g} / \mathrm{ml} \pm 0.28 \mu \mathrm{g} / \mathrm{ml}$, and the resistance index was 5.2 (Figure $4(\mathrm{~d})$ ). The IC50 of cisplatin in UCA1 siRNA group was significantly lower than that in NC group $(t=17.51, P<0.0001)$, control group $(t=$ $37.65, P<0.0001)$. The IC50 of A549/DDP cells decreased from $10.7 \mu \mathrm{g} / \mathrm{ml} \pm 0.28 \mu \mathrm{g} / \mathrm{ml}$ to $3.6 \mu \mathrm{g} / \mathrm{ml} \pm 0.12 \mu \mathrm{g} / \mathrm{ml}$ after UCA1 knockdown, as shown in Figure 4(f). The results showed that UCA1 siRNA, A549/DDP cisplatin resistance can be significantly reversed.

3.9. UCA1 Overexpression Significantly Increased the IC50 of Cisplatin in NCI-H1299 Cell. The results showed that the IC50 of cisplatin in UCA1 OE group was significantly higher than that in NC group $(t=23.21, P<0.0001)$, control group $(t=29.34, P<0.0001)$. The IC50 of NCI-H1299 cells increased from $1.20 \mu \mathrm{g} / \mathrm{ml} \pm 0.04 \mu \mathrm{g} / \mathrm{ml}$ to $4.5 \mu \mathrm{g} / \mathrm{ml} \pm$ $0.13 \mu \mathrm{g} / \mathrm{ml}$ after UCA1 was overexpressed, as shown in Figure 4(f). The results showed that UCA1 was overexpressed; NCI-H1299 cisplatin resistance can be significantly increased.

\section{Discussion}

LncRNAs are involved in many biological processes, as Xchromosome inactivation, gene imprinting [33, 34]. Otherwise, lncRNAs are important factors in the control of gene 


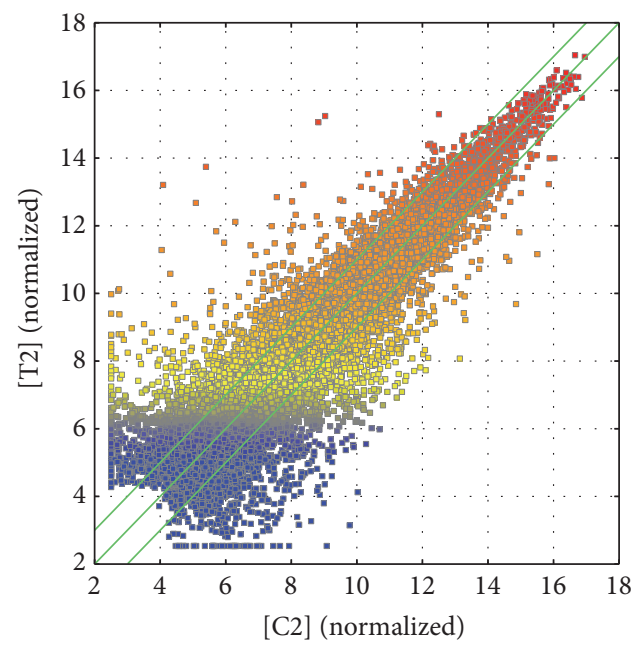

(a)

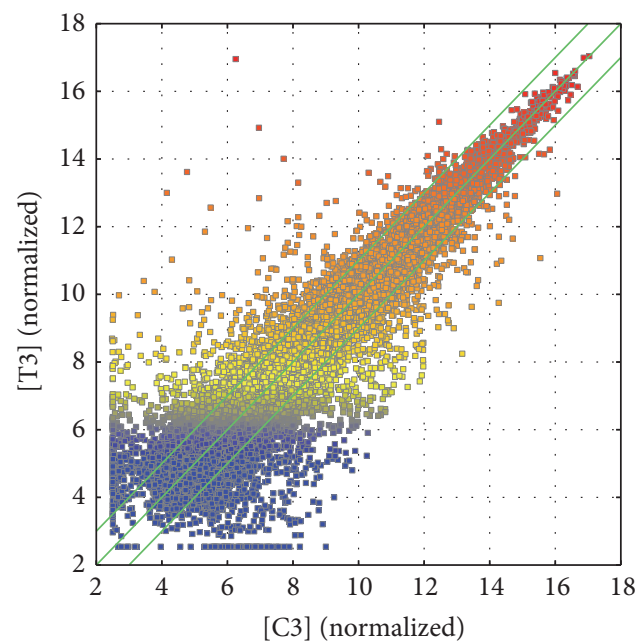

(b)

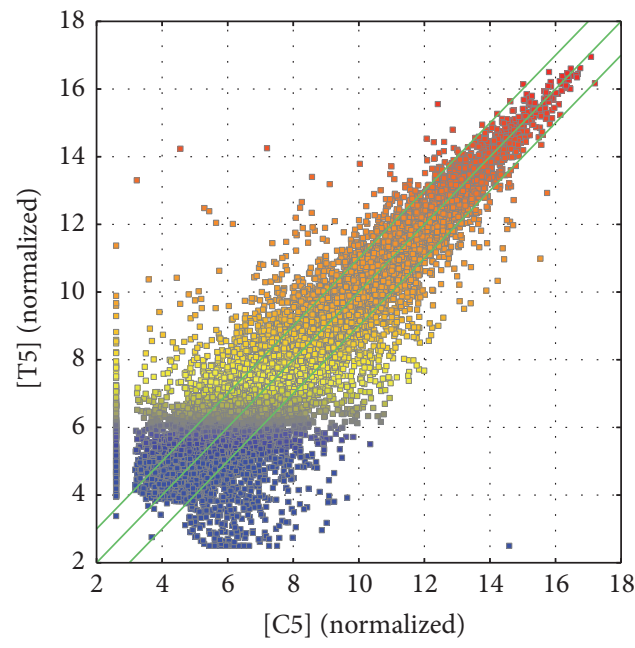

(c)

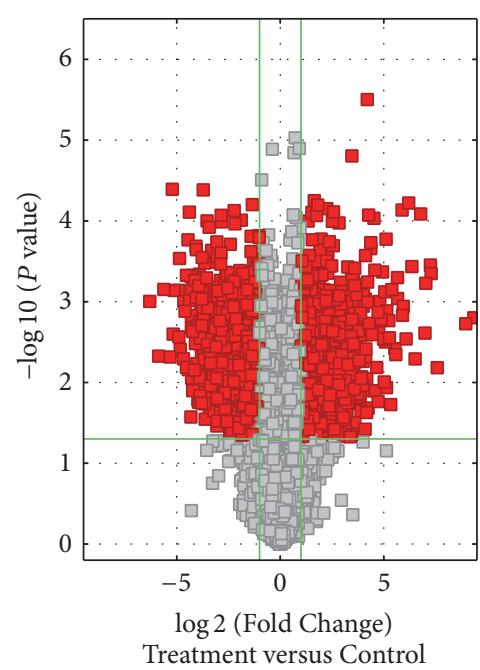

(d)

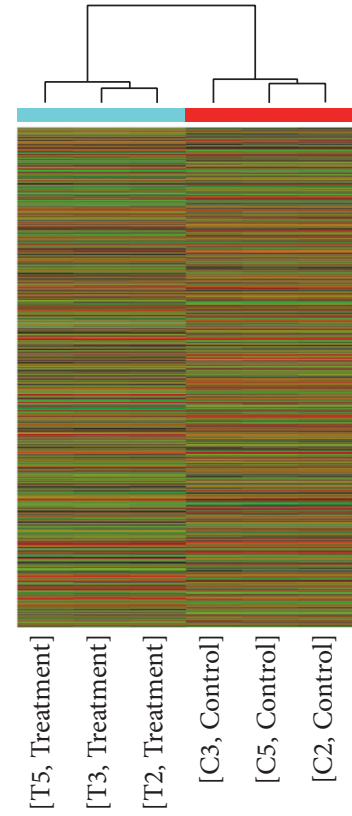

(e)

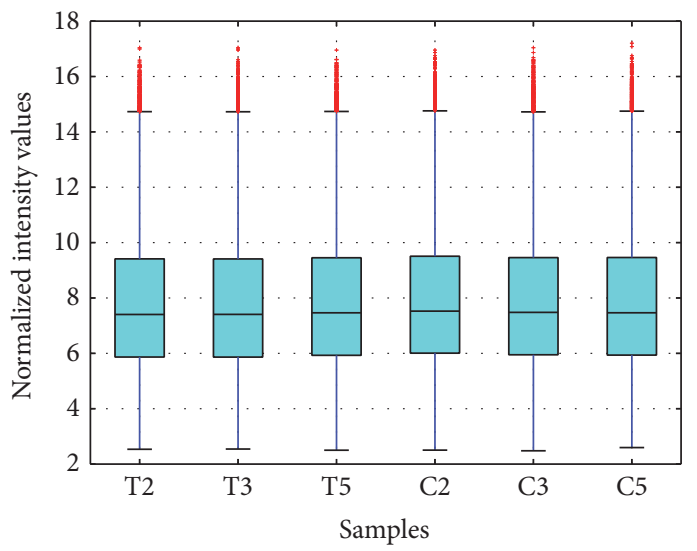

(f)

FIGURE 2: Box plots, scatter plots, and heat map showing the variation in mRNA expression between the A549/DDP and A549 arrays. The values of the $X$ and $Y$ axes in the scatter plot are averaged normalized values in each group $(\log 2$-scaled). The mRNAs above the top green line and below the bottom green line are those with a $>3$-fold change in expression between tissues. (a) Scatter plots of T2 group versus C2 group, (b) Scatter plots of T3 group versus C3 group. (c) Scatter plots of T5 group versus C5 group. (d) Volcanic map. (e) Heat map and hierarchical clustering of lncRNA. (f) Box plots showing the distribution of the lncRNA. 


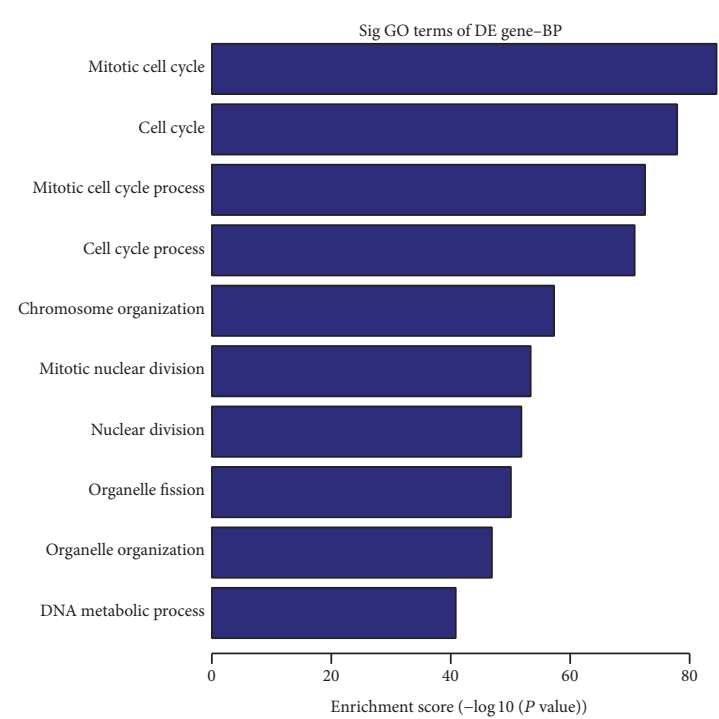

(a)

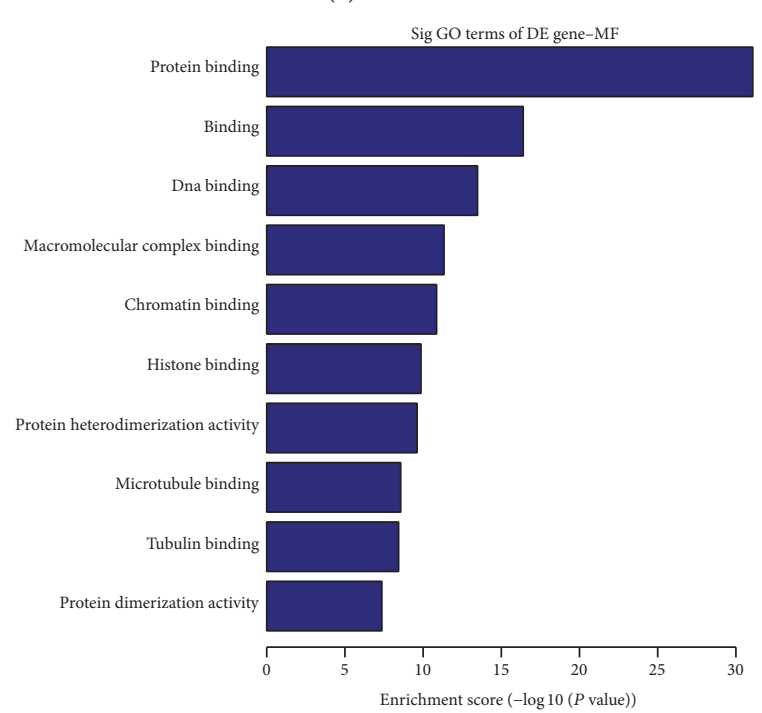

(c)

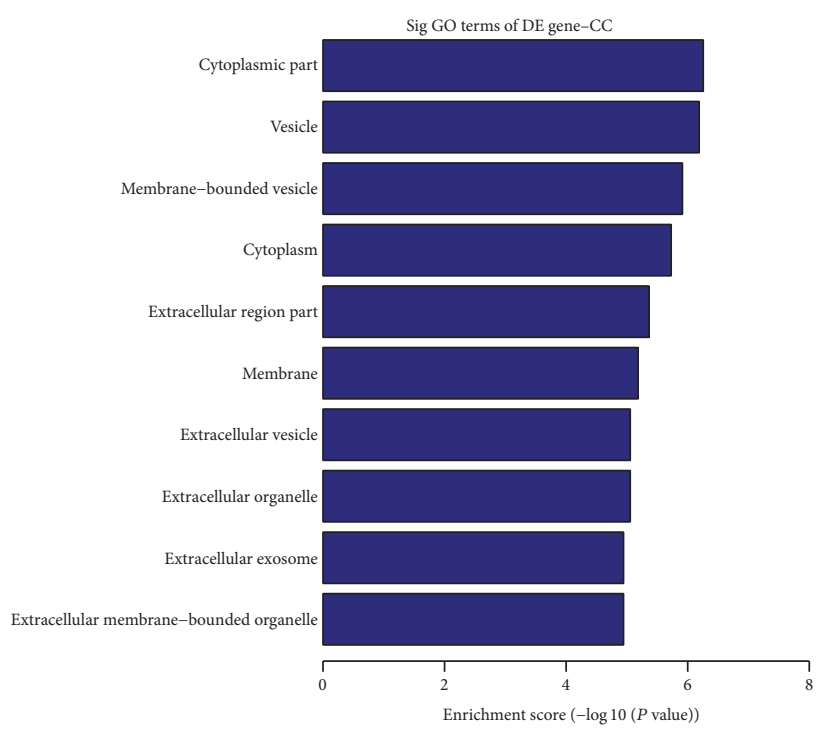

(e)

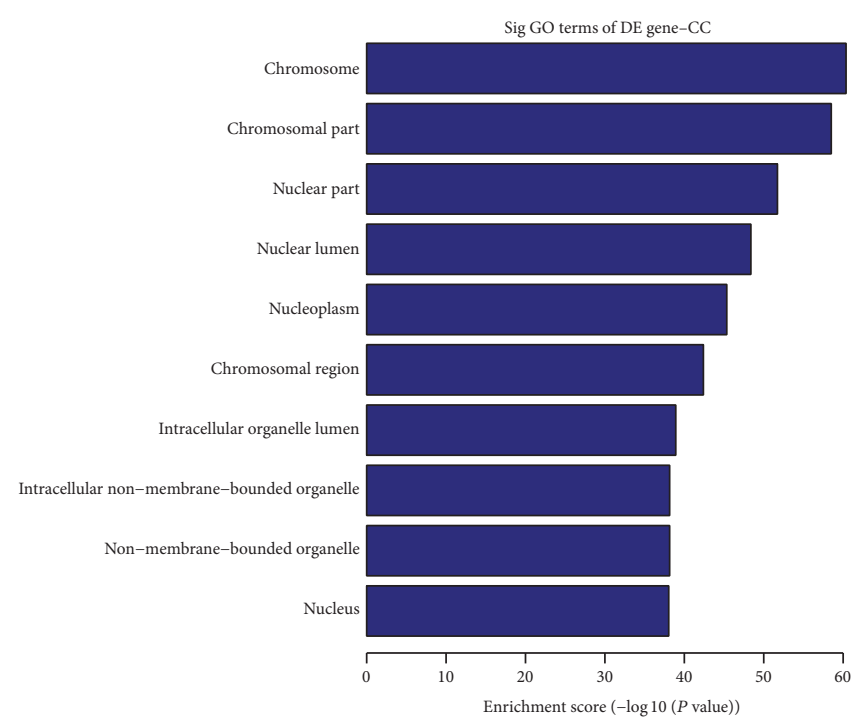

(b)

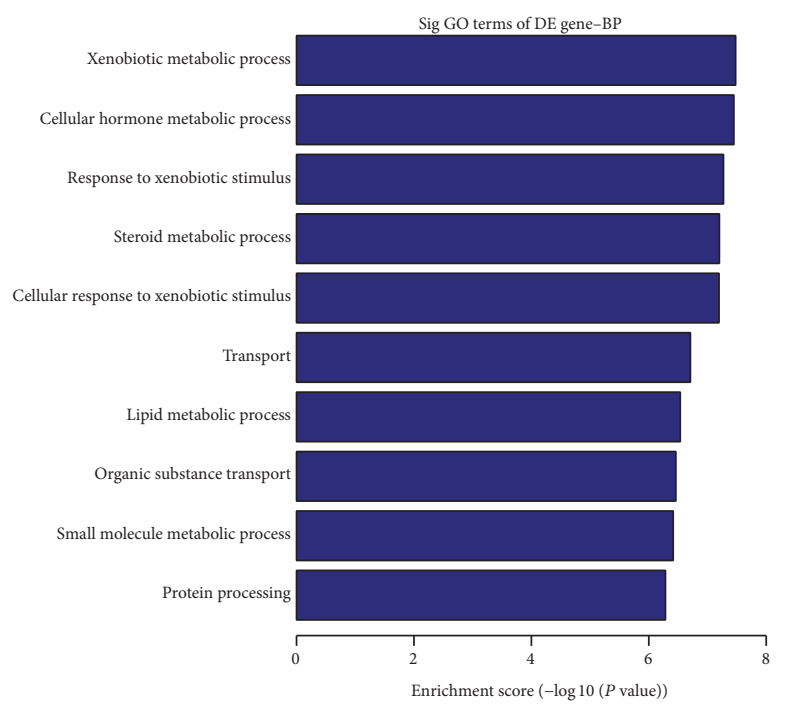

(d)

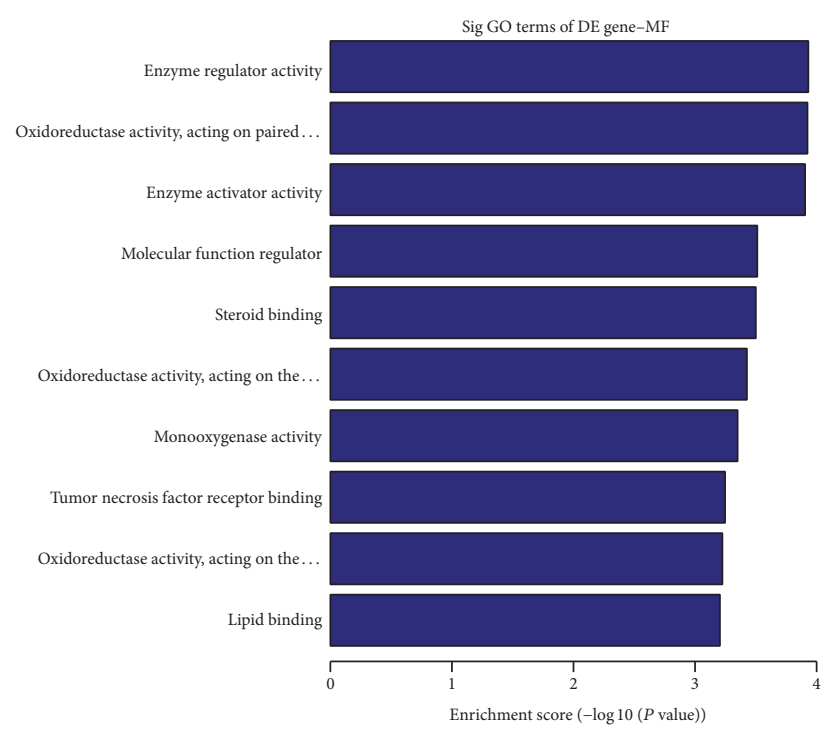

(f)

FIgURE 3: Continued. 


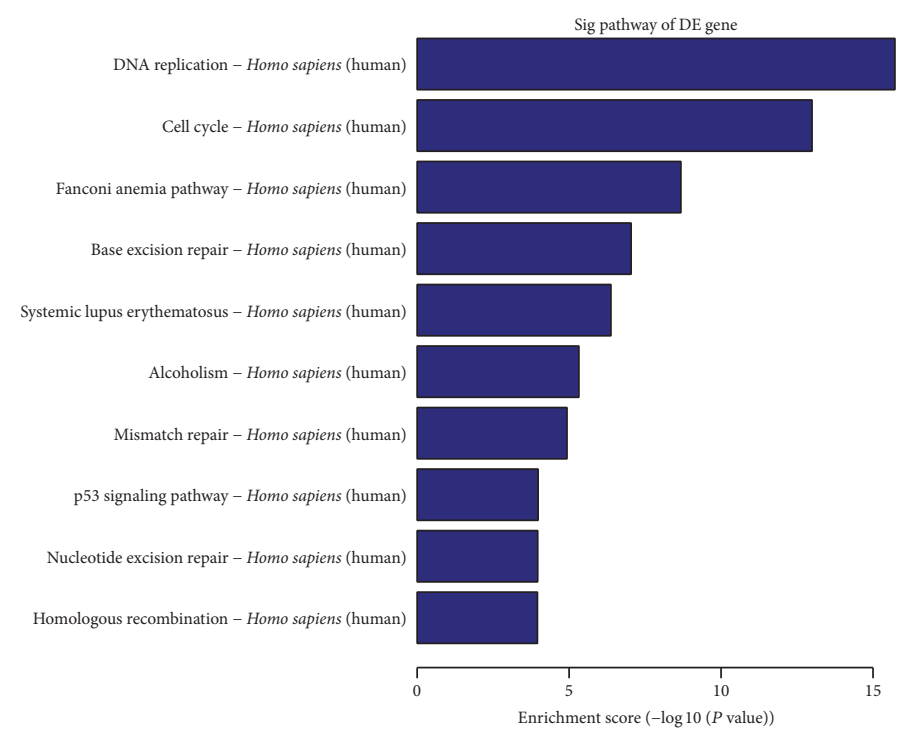

(g)

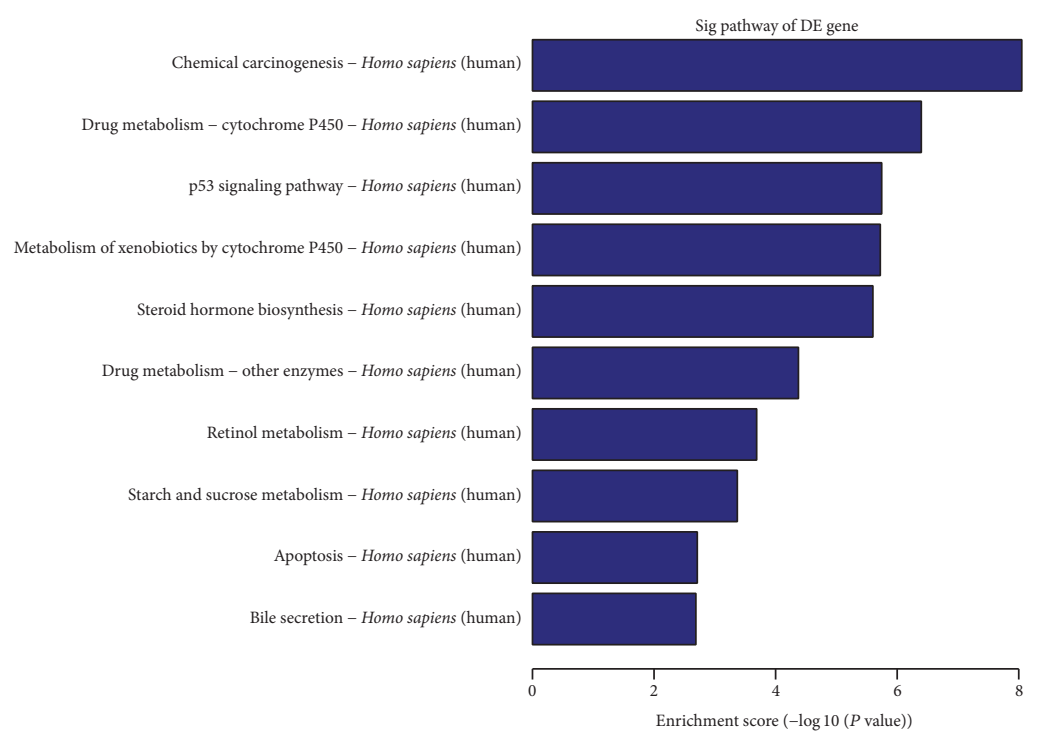

(h)

FIGURE 3: GO and pathway analysis of mRNA from cisplatin resistance in lung adenocarcinoma. (a) Biological processes of downregulated mRNA. (b) Cellular components of downregulated mRNA. (c) Molecular functions of downregulated mRNA. (d) Biological processes of upregulated mRNA. (e) Cellular components of upregulated mRNA. (f) Molecular functions of upregulated mRNA. (g) Pathway analysis of downregulated mRNA from cisplatin resistance in lung adenocarcinoma. (h) Pathway analysis of upregulated mRNA from cisplatin resistance in lung adenocarcinoma.

expression in tumor [35] and play an important role in the development, progression, and drug resistance of tumors [36]. Recently, disease-lncRNA association prediction is a recent trend for the identifying potential disease-related lncRNAs [37, 38]. Developing powerful computational models for potential disease-related lncRNAs identification would benefit biomarker identification and drug discovery for human disease diagnosis, treatment, prognosis, and prevention $[37,38]$.

In this study, we analyzed lncRNA abnormal expression profiles and ascertained the potential role of cisplatin resistance in LAD. High-throughput microarray techniques revealed a variety of differentially expressed lncRNAs, including 984 lncRNAs upregulated and 559 lncRNAs downregulated in A549/DDP cell compared to A549 cell. LncRNAs are usually divided into five categories: sense, antisense, bidirectional, intronic, and intergenic. LncRNAs are known to function by a variety of mechanisms. However, a common and important function of lncRNAs is to change the expression of nearby mRNAs by influencing process of transcription [39] or directly playing an enhancer-like role $[40,41]$. In the study, we increased the accuracy of target prediction by comparing 


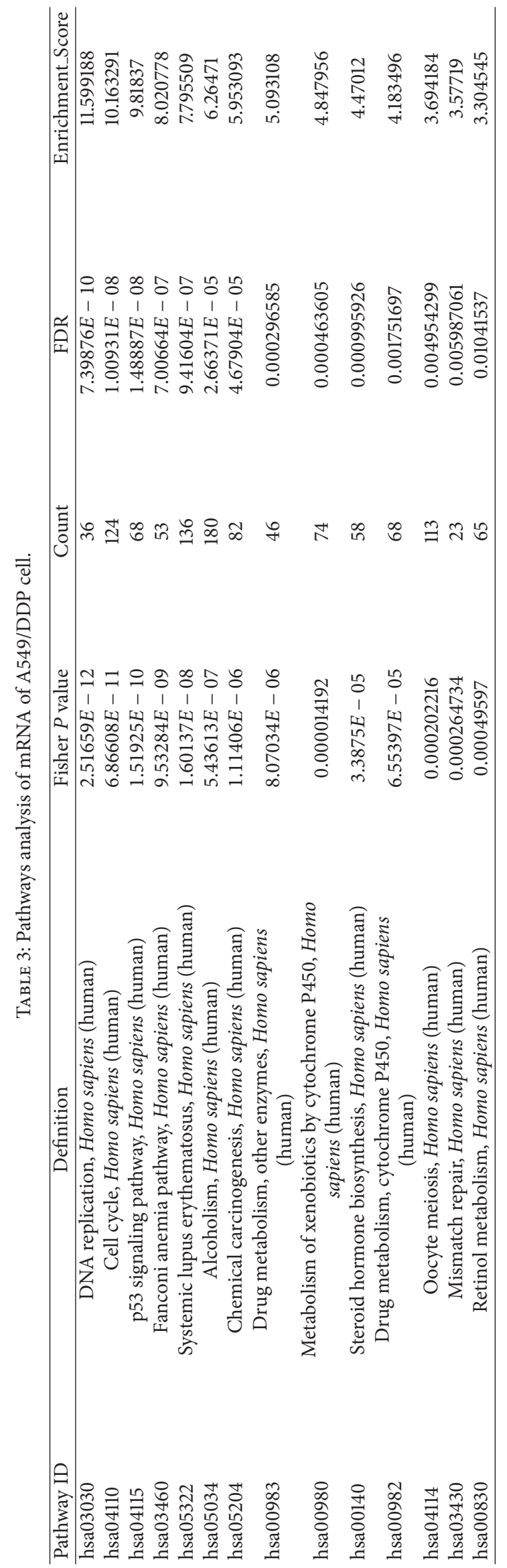




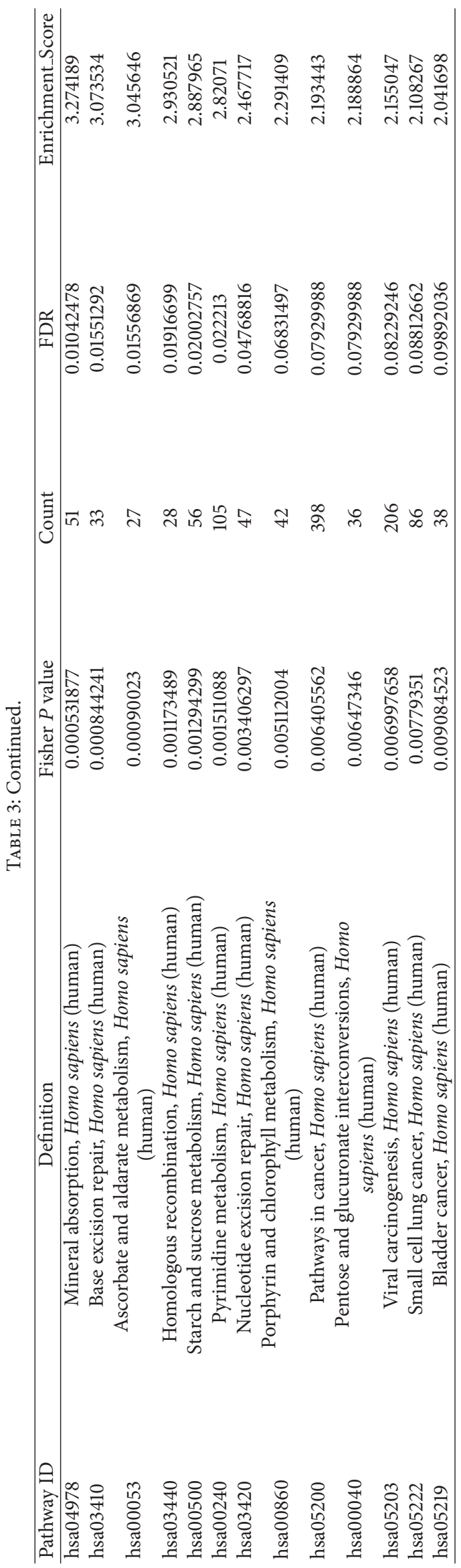




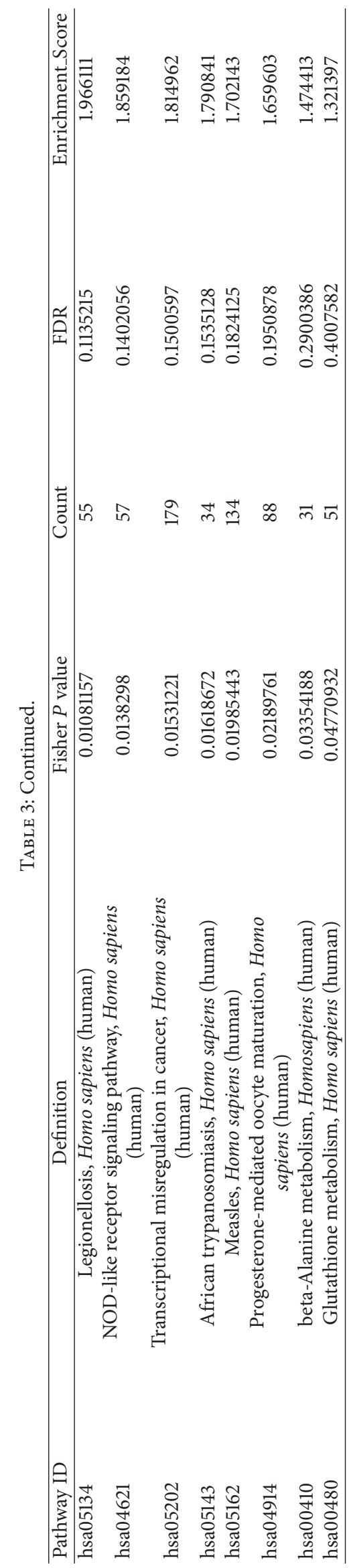




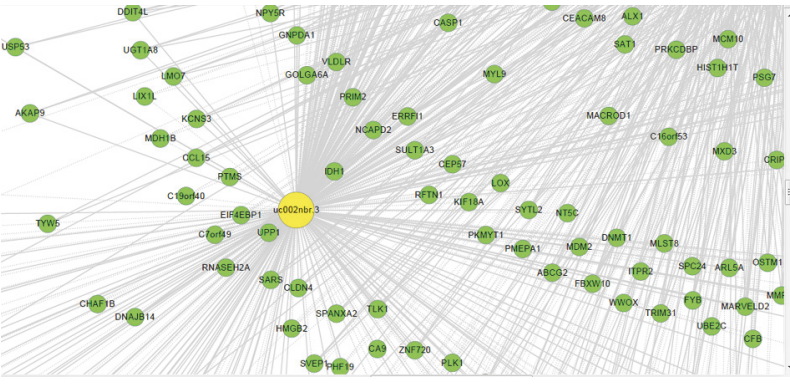

(a)

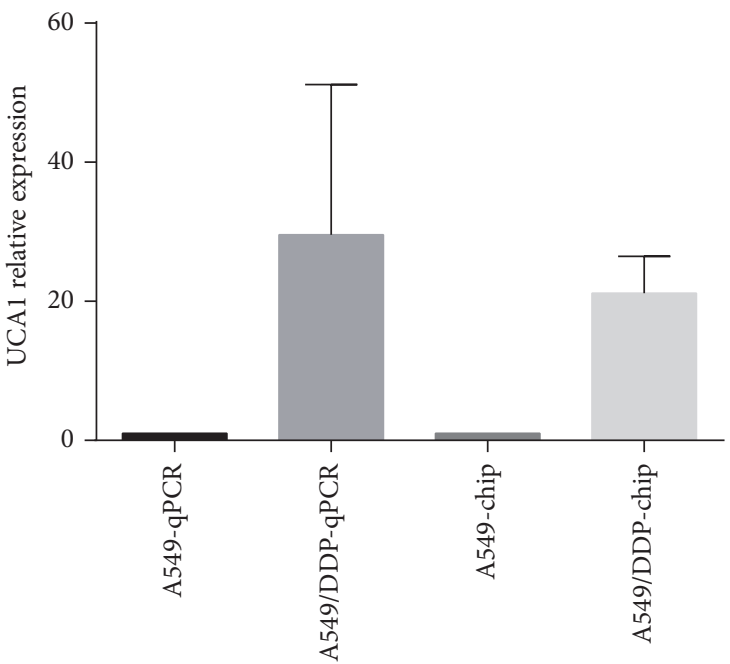

(c)

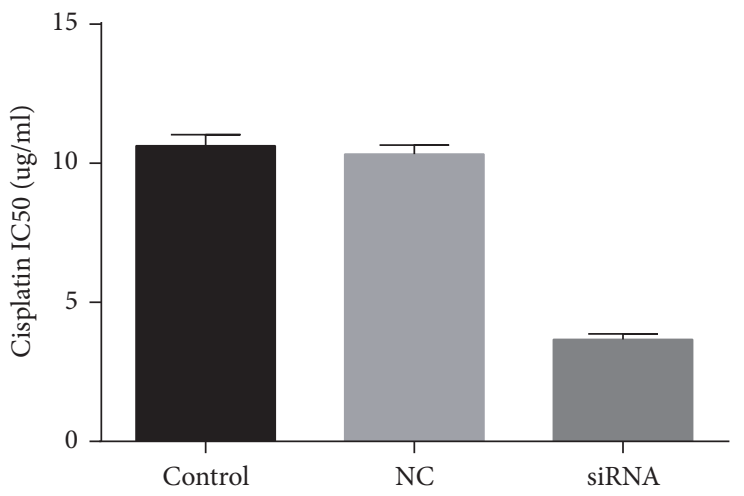

(e)

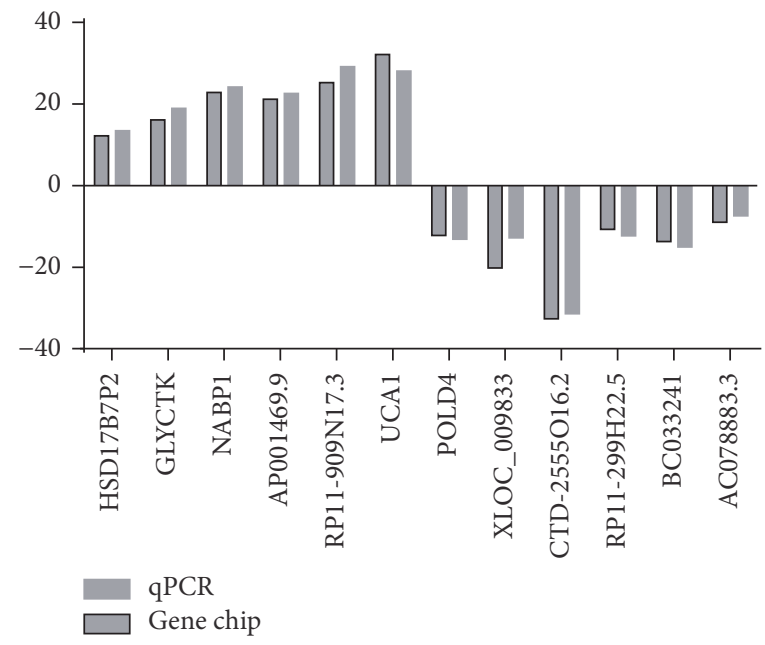

(b)

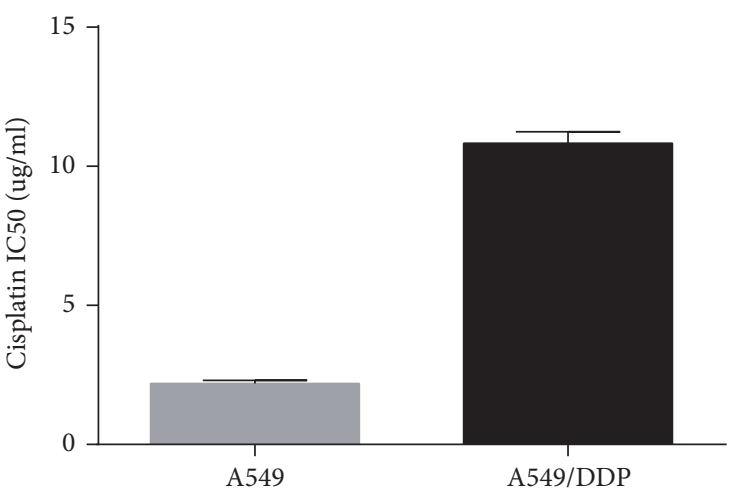

(d)

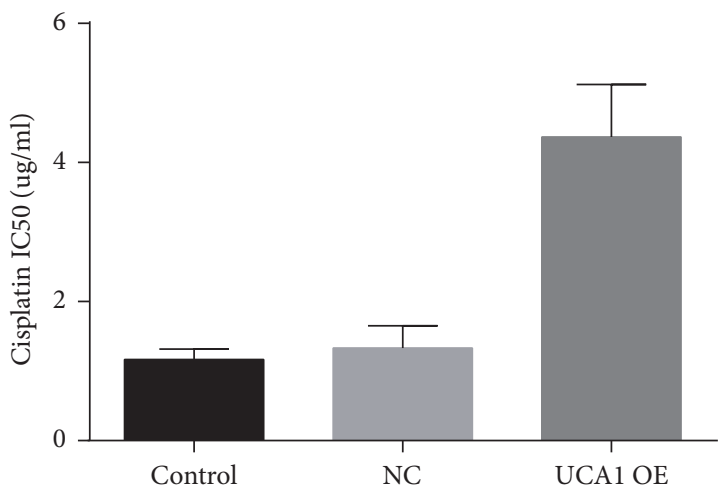

(f)

FIGURE 4: Some lncRNA expression in LAD and A549/DDP, A549 cell. (a) LncRNA-mRNA coexpression network, the results imply that UCA1, ENST00000443252, ENST00000510562, ENST00000565689, ENST00000558690, ENST00000397340, ENST00000440955, and ENST00000507916 are also closely related to many mRNA molecules. (b) Comparison between gene chip data and qPCR result. The validation results of the 12 lncRNAs indicated that the microarray data correlated well with the GPCR results. UCA1 expression level in LAD and A549/DDP, A549 cell. (c) The expression of UCA1 in cisplatin-resistant A549/DDP cells was significantly higher than that in cisplatin-sensitive A549 cells. (d) We used CCK-8 method to detect the sensitivity of A549/DDP cells to cisplatin. (e) The IC50 of cisplatin in UCA1 siRNA group was significantly lower than that in NC group, control group. (f) The IC50 of cisplatin in UCA1 overexpression group was significantly higher than that in NC group, control group. 
abnormally expressed mRNAs with differentially expressed lncRNAs. The expression profiles of 43 intergenic lncRNAs (lincRNAs) hinted that they were differentially expressed between A549/DDP cell and A549 cell. Among these, 31 were upregulated and 12 were downregulated. The expression profiles of 33 lncRNA-a indicated that they were differentially expressed between A549/DDP and A549 cell. Among these, 17 were upregulated and 16 were downregulated. Otherwise, we found 52 antisense lncRNAs, among these, 21 were upregulated and 31 were downregulated. So as to get insights into lncRNA target gene function, GO analysis and KEGG pathway annotation were applied to the lncRNA target gene pool. GO analysis uncovered that the number of genes corresponding to downregulated mRNAs was larger than that relating to upregulated mRNAs. KEGG annotation unveiled that there were 30 upregulated pathways (including chemical carcinogenesis, drug metabolism, and p53 signaling pathway) and 37 downregulated pathways (including DNA replication, cell cycle, and Fanconi anemia pathway). These pathways might be involved in the occurrence and development of cisplatin resistance in LAD.

We found that 12 of the lncRNAs identified in the microarray analysis were confirmed by qPCR to be aberrantly expressed in A549/DDP cell. Among these lncRNAs, UCA1 was the significantly upregulated. Furthermore, we built the lncRNA-mRNA coexpression network and it is shown that UCA1 and some lncRNAs were individually related to some mRNAs; it hinted that lncRNA-mRNA coexpression network might contribute to the development of cisplatin resistance in LAD. UCA1 has also been reported to be related to cisplatin resistance in bladder carcinoma $[42,43]$. The expression of UCA1 in A549/DDP cells was significantly higher than that in A549 cells, suggesting that UCA1 may play an important role in cisplatin resistance in LAD. Subsequently, we found the IC50 of A549/DDP cells decreased from $10.7 \mu \mathrm{g} / \mathrm{ml} \pm$ $0.28 \mu \mathrm{g} / \mathrm{ml}$ to $3.6 \mu \mathrm{g} / \mathrm{ml} \pm 0.12 \mu \mathrm{g} / \mathrm{ml}$ after UCA1 knockdown, while the IC50 of NCI-H1299 cells increased from $1.20 \mu \mathrm{g} / \mathrm{ml}$ $\pm 0.04 \mu \mathrm{g} / \mathrm{ml}$ to $4.5 \mu \mathrm{g} / \mathrm{ml} \pm 0.13 \mu \mathrm{g} / \mathrm{ml}$ after UCA1 was overexpressed; it hinted that UCA1 might contribute to the development of cisplatin resistance in LAD and further study of the biological function of UCA1 will be required to confirm this notion.

Our study revealed a set of lncRNAs with differential expression from cisplatin resistance in LAD. Furthermore, potential roles for these lncRNAs in the regulation of chemical carcinogenesis and DNA replication signaling pathways were identified. Moreover, we found that UCA1 might contribute to the development of cisplatin resistance in LAD.

\section{Conflicts of Interest}

The authors declare that no conflicts of interest exist.

\section{Authors' Contributions}

Lijuan $\mathrm{Hu}$ and Jian Chen contributed equally to this work.

\section{Acknowledgments}

This study was financially supported by the National Natural Science Foundation of China (8140736, 81672088), Zhejiang
Provincial Natural Science Foundation (LQ16H160020), the Key Construction Academic Subject (medical innovation) of Zhejiang Province (treatment of critical trauma discipline), and the Wenzhou Municipal Science and Technology Bureau, China (Y20160160, Y20170208).

\section{References}

[1] J.-P. Pignon, H. Tribodet, G. V. Scagliotti et al., "Lung adjuvant cisplatin evaluation: a pooled analysis by the LACE collaborative group," Journal of Clinical Oncology, vol. 26, no. 21, pp. 35523559, 2008.

[2] K. Zarogoulidis, P. Zarogoulidis, K. Darwiche et al., "Treatment of non-small cell lung cancer (NSCLC)," Journal of Thoracic Disease, vol. 5, Suppl 4, pp. S389-S396, 2013.

[3] P. A. Bunn and K. Kelly, "New combinations in the treatment of lung cancer: a time for optimism," Chest, vol. 117, no. 4, pp. 138S-143S, 2000.

[4] S. C. Spiro and G. A. Silvestri, "One hundred years of lung cancer," American Journal of Respiratory and Critical Care Medicine, vol. 172, no. 5, pp. 523-529, 2005.

[5] M.-K. Choi and D.-D. Kim, "Platinum transporters and drug resistance," Archives of Pharmacal Research, vol. 29, no. 12, pp. 1067-1073, 2006.

[6] L. P. Martin, T. C. Hamilton, and R. J. Schilder, "Platinum resistance: the role of DNA repair pathways," Clinical Cancer Research, vol. 14, no. 5, pp. 1291-1295, 2008.

[7] M. Wangpaichitr, C. Wu, M. You et al., "Inhibition of mTOR restores cisplatin sensitivity through down-regulation of growth and anti-apoptotic proteins," European Journal of Pharmacology, vol. 591, no. 1-3, pp. 124-127, 2008.

[8] P. Sève and C. Dumontet, "Chemoresistance in non-small cell lung cancer," Current Medicinal Chemistry-Anti-Cancer Agents, vol. 5, no. 1, pp. 73-88, 2005.

[9] M. Ohmichi, J. Hayakawa, K. Tasaka, H. Kurachi, and Y. Murata, "Mechanisms of platinum drug resistance," Trends in Pharmacological Sciences, vol. 26, no. 3, pp. 113-116, 2005.

[10] C. Wu, M. Wangpaichitr, L. Feun et al., "Overcoming cisplatin resistance by mTOR inhibitor in lung cancer," Molecular Cancer, vol. 4, no. 1, p. 25, 2005.

[11] M. Zhou, Z. Zhang, H. Zhao, S. Bao, L. Cheng, and J. Sun, "An immune-related six-lncrna signature to improve prognosis prediction of glioblastoma multiforme," Molecular Neurobiology, pp. 1-14, 2017.

[12] M. Zhou, Z. Diao, X. Yue et al., "Construction and analysis of dysregulated lncRNA-associated ceRNA network identified novel lncRNA biomarkers for early diagnosis of human pancreatic cancer," Oncotarget, vol. 7, no. 35, pp. 56383-56394, 2016.

[13] M. Zhou, H. Zhao, W. Xu, S. Bao, L. Cheng, and J. Sun, "Discovery and validation of immune-associated long noncoding RNA biomarkers associated with clinically molecular subtype and prognosis in diffuse large B cell lymphoma," Molecular Cancer, vol. 16, no. 1, article no. 16, 2017.

[14] W. Guo, Q. Wang, Y. Zhan et al., "Transcriptome sequencing uncovers a three-long noncoding RNA signature in predicting breast cancer survival," Scientific Reports, vol. 6, Article ID 27931, 2016.

[15] M. Zhou, Y. Sun, Y. Sun et al., "Comprehensive analysis of lncRNA expression profiles reveals a novel lncRNA signature to discriminate nonequivalent outcomes in patients with ovarian cancer," Oncotarget , vol. 7, no. 22, pp. 32433-32448, 2016. 
[16] Z. Tu, D. He, X. Deng et al., "An eight-long non-coding RNA signature as a candidate prognostic biomarker for lung cancer," Oncology Reports, vol. 36, no. 1, pp. 215-222, 2016.

[17] M. Zhou, X. Wang, H. Shi et al., "Characterization of long non-coding RNA-associated ceRNA network to reveal potential prognostic lncRNA biomarkers in human ovarian cancer," Oncotarget, vol. 7, no. 11, pp. 12598-12611, 2016.

[18] M. Zhou, H. Zhao, Z. Wang et al., "Identification and validation of potential prognostic lncRNA biomarkers for predicting survival in patients with multiple myeloma," Journal of Experimental \& Clinical Cancer Research, vol. 34, no. 102, 2015.

[19] M. Zhou, M. Guo, D. He et al., "A potential signature of eight long non-coding RNAs predicts survival in patients with nonsmall cell lung cancer," Journal of Translational Medicine, vol. 13, no. 1, article no. 231, 2015.

[20] H. Zhang, Z. Chen, X. Wang et al., "Long non-coding RNA: a new player in cancer," Journal of hematology oncology, vol. 6, no. 37, 2013.

[21] N. Hauptman and D. Glavač, "Long non-coding RNA in cancer," International Journal of Molecular Sciences, vol. 14, no. 3, pp. 4655-4669, 2013.

[22] G. Chen, Z. Wang, D. Wang et al., "LncRNADisease: a database for long-non-coding RNA-associated diseases," Nucleic Acids Research, vol. 41, no. 1, pp. D983-D986, 2013.

[23] J. Liu, L. Wan, K. Lu et al., “The long noncoding RNA MEG3 contributes to cisplatin resistance of human lung adenocarcinoma," PLoS ONE, vol. 10, no. 5, Article ID e0114586, 2015.

[24] X.-W. Zhang, P. Bu, L. Liu, X.-Z. Zhang, and J. Li, "Overexpression of long non-coding RNA PVT1 in gastric cancer cells promotes the development of multidrug resistance," Biochemical and Biophysical Research Communications, vol. 462, no. 3, pp. 227-232, 2015.

[25] Q. Hang, R. Sun, C. Jiang, and Y. Li, "Notch 1 promotes cisplatinresistant gastric cancer formation by upregulating lncRNA AK022798 expression," Anti-Cancer Drugs, vol. 26, no. 6, pp. 632-640, 2015.

[26] Y. Fan, B. Shen, M. Tan et al., "Long non-coding RNA UCA1 increases chemoresistance of bladder cancer cells by regulating Wnt signaling," FEBS Journal, vol. 281, no. 7, pp. 1750-1758, 2014.

[27] Z. Liu, M. Sun, K. Lu et al., “The long noncoding RNA HOTAIR contributes to cisplatin resistance of human lung adenocarcinoma cells via downregualtion of p21WAF1/CIP1 expression," PLoS ONE, vol. 8, no. 10, Article ID e77293, 2013.

[28] Y. Yang, H. Li, S. Hou, B. Hu, J. Liu, and J. Wang, " The noncoding RNA expression profile and the effect of lncRNA AK126698 on cisplatin resistance in non-small-cell lung cancer cell," PLoS ONE, vol. 8, no. 5, Article ID e65309, 2013.

[29] Q. Wang, N. Cheng, X. Li et al., "Correlation of long non-coding RNA H19 expression with cisplatin-resistance and clinical outcome in lung adenocarcinoma," Oncotarget, vol. 8, no. 2, pp. 2558-2567, 2017.

[30] H. Shi, J. Pu, X. Zhou, Y. Ning, and C. Bai, "Silencing long noncoding RNA ROR improves sensitivity of non-small-cell lung cancer to cisplatin resistance by inhibiting PI3K/Akt/mTOR signaling pathway," Tumour biology: The Journal of The International Society for Oncodevelopmental Biology and Medicine, vol. 39, article 1010428317697568, no. 5, p. 101042831769756, 2017.

[31] G. Xu, J. Chen, and Q. Pan, "Long noncoding RNA expression profiles of lung adenocarcinoma ascertained by microarray analysis," PLoS ONE, vol. 9, no. 8, Article ID e104044, 2014.
[32] S. Ren, Z. Peng, J. Mao et al., "RNA-seq analysis of prostate cancer in the Chinese population identifies recurrent gene fusions, cancer-associated long noncoding RNAs and aberrant alternative splicings," Cell Research, vol. 22, no. 5, pp. 806-821, 2012.

[33] T. R. Mercer, M. E. Dinger, and J. S. Mattick, "Long non-coding RNAs: insights into functions," Nature Reviews Genetics, vol. 10, no. 3, pp. 155-159, 2009.

[34] K. C. Wang and H. Y. Chang, "Molecular mechanisms of long noncoding RNAs," Molecular Cell, vol. 43, no. 6, pp. 904-914, 2011.

[35] A. N. Khachane and P. M. Harrison, "Mining mammalian transcript data for functional long non-coding RNAs," PLoS ONE, vol. 5, no. 4, Article ID e10316, 2010.

[36] R. A. Gupta, N. Shah, K. C. Wang et al., "Long non-coding RNA HOTAIR reprograms chromatin state to promote cancer metastasis," Nature, vol. 464, no. 7291, pp. 1071-1076, 2010.

[37] X. Chen, "Predicting lncRNA-disease associations and constructing lncRNA functional similarity network based on the information of miRNA," Scientific Reports, vol. 5, Article ID 13186, 2015.

[38] X. Chen and G.-Y. Yan, "Novel human lncRNA-disease association inference based on lncRNA expression profiles," Bioinformatics, vol. 29, no. 20, pp. 2617-2624, 2013.

[39] J. S. Mattick and M. J. Gagen, “The evolution of controlled multitasked gene networks: The role of introns and other noncoding RNAs in the development of complex organisms," Molecular Biology and Evolution, vol. 18, no. 9, pp. 1611-1630, 2001.

[40] U. A. Ørom, T. Derrien, M. Beringer et al., "Long noncoding RNAs with enhancer-like function in human cells," Cell, vol. 143, no. 1, pp. 46-58, 2010.

[41] J. S. Mattick, "Linc-ing long noncoding RNAs and enhancer function," Developmental Cell, vol. 19, no. 4, pp. 485-486, 2010.

[42] M. A. Yemul, "A flail anterior tricuspid leaflet secondary to anteroposterior papillary muscle rupture in a neonate," Journal of Echocardiography, vol. 31, no. 5, pp. E151-E155, 2014.

[43] J. Pan, X. Li, W. Wu et al., "Long non-coding RNA UCA1 promotes cisplatin/gemcitabine resistance through CREB modulating miR-196a-5p in bladder cancer cells," Cancer Letters, vol. 382, no. 1, pp. 64-76, 2016. 

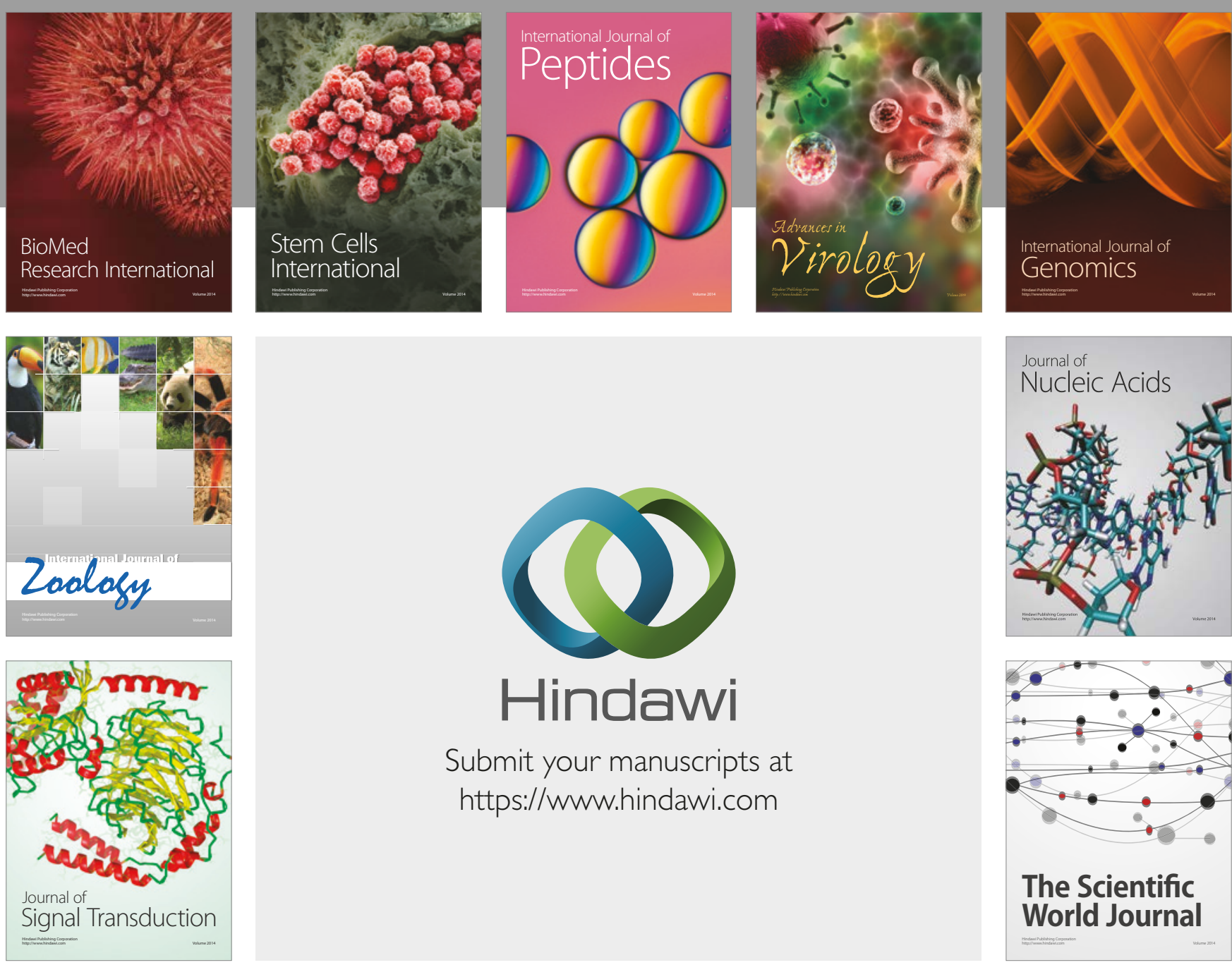

Submit your manuscripts at

https://www.hindawi.com
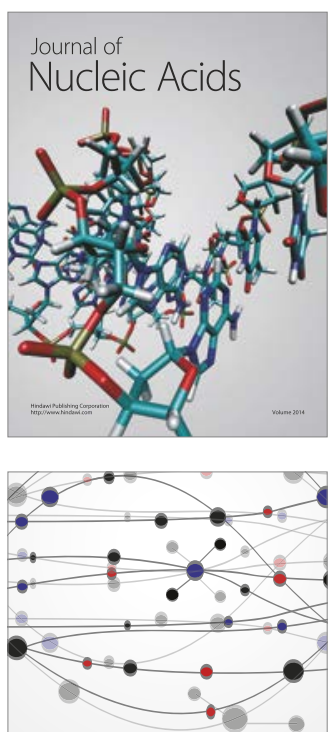

The Scientific World Journal

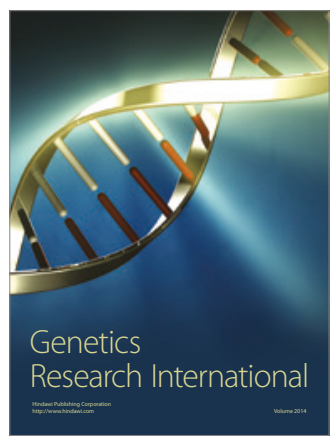

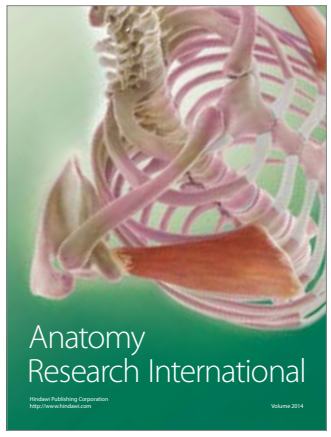

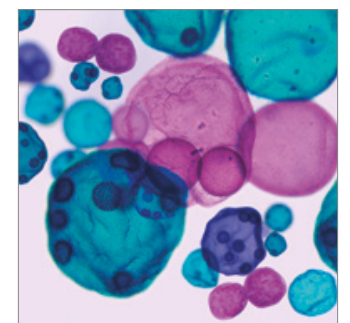

International Journal of Microbiology
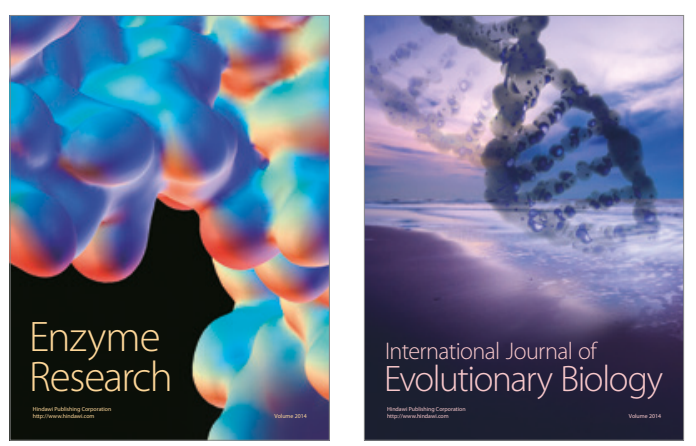
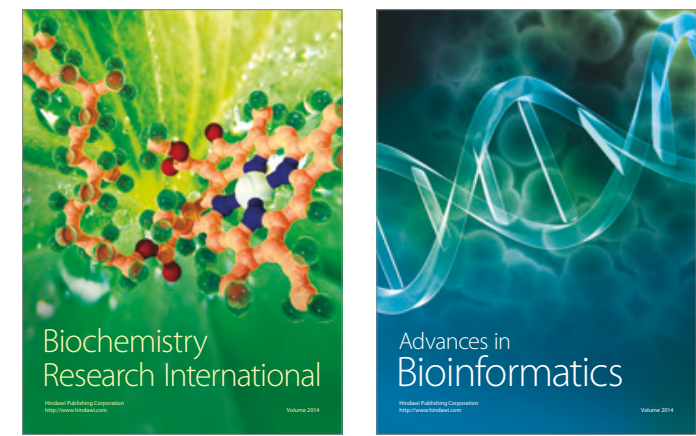

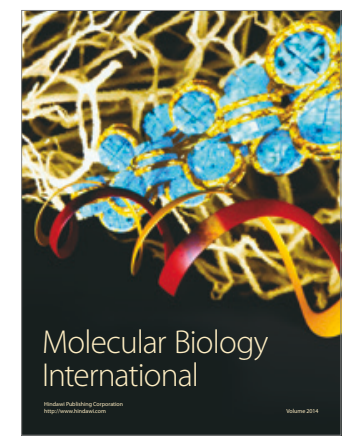

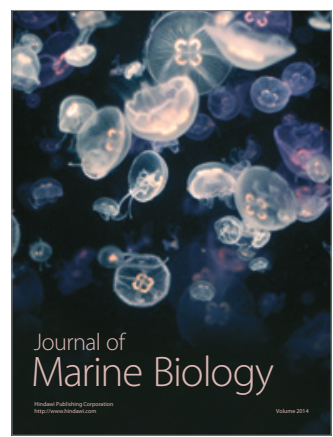

